\title{
Involvement of TL1A and DR3 in induction of ischaemia and inflammation in urinary bladder dysfunction in the elderly
}

\author{
WEI WANG ${ }^{1}$, NING ZHANG ${ }^{1}$, XU-HUI ZHU ${ }^{1}$, ZHI-SONG HE ${ }^{2}$, \\ WASILIJIANG WAHAFU ${ }^{2}$, ZHI-QING $\mathrm{XU}^{3}$ and YONG YANG ${ }^{1}$ \\ ${ }^{1}$ Urology Department, Beijing Chao-Yang Hospital, Capital Medical University, Beijing 100020; ${ }^{2}$ Department of Urology, \\ Peking University First Hospital, The Institute of Urology, Peking University, Beijing 100034; \\ ${ }^{3}$ Beijing Institute of Neuroscience, Beijing Key Laboratory of Neural Regeneration and Repair, \\ Capital Medical University, Beijing 100069, P.R. China
}

Received January 31, 2012; Accepted May 11, 2012

DOI: $10.3892 / \mathrm{mmr} .2012 .928$

\begin{abstract}
Benign prostatic hyperplasia often causes intravesical obstruction in elderly men; however, the processes of aging and bladder outlet obstruction independently evoke alterations in the structure and function of the bladder. These changes lead to lower urinary tract symptoms; however, it is difficult to separate the effects of prostatic obstruction on the bladder from those of aging. Nevertheless, few studies have focused on elucidating the pathophysiological mechanisms in the aged bladder. Bladder dysfunction due to detrusor instabitliy (caused by old age) is considered to be associated with chronic ischaemia and inflammation. The aim of this study was to explore the role of tumour necrosis factor (TNF)-like ligand 1A (TL1A) and death-domain receptor (DR)3 in the ischaemic and inflammatory process of aged bladder dysfunction. Sixteen bladder tissue samples collected from patients with urothelial tumours of the bladder were divided into two groups according to age: group 1 (controls, $n=8$ ) and group 2 (aged group, $n=8$ ). Urodynamic examinations were preformed before radical cystectomy. The full-thickness bladder tissues were obtained at least $5 \mathrm{~cm}$ away from the margin of the tumours. The mRNA expression levels of TL1A, DR3, von Willebrand factor (vWF), interleukin (IL)-6 and nerve growth factor (NGF) in the two groups were determined using
\end{abstract}

Correspondence to: Professor Yong Yang, Urology Department, Beijing Chao-Yang Hospital, Capital Medical University, No. 8 Gong Ti Nan Lu, Chaoyang District, Beijing 100020, P.R. China E-mail: yoya@china.com

Dr Zhi-Qing Xu, Beijing Institute of Neuroscience, Beijing Key Laboratory of Neural Regeneration and Repair, Capital University of Medical Sciences, 10 Xitoutiao, Youanmen, Fengtai District, Beijing 100069, P.R. China

E-mail: zhiqingx@ccmu.edu.cn

Key words: tumour necrosis factor-like ligand 1A, vascular endothelial cell growth inhibitor, death-domain receptor 3, bladder ischaemia, lower urinary tract symptoms, aging bladder real-time reverse transcription-PCR and the protein expression levels of TL1A, DR3 and p65 were determined by western blot analysis. The TL1A and DR3 mRNA and protein expression levels of the aged bladders were upregulated compared to the control group $(\mathrm{p}<0.05)$. Compared to the control, the mRNA expression levels of $\mathrm{vWF}$ in the aged bladder tissues were markedly lower $(\mathrm{p}<0.01)$; however, the mRNA expression levels of IL- 6 were significantly higher in the aged bladder tissues $(\mathrm{p}<0.01)$ compared to the control. No significant difference in NGF mRNA expression between the two groups was detected ( $p>0.05$ ). In conclusion, the aged bladder was associated with ischaemic and inflammatory alterations in comparison to the control group. TL1A and DR3 may play an important role in the pathophysiological process of the aged bladder.

\section{Introduction}

A variety of changes in bladder function have been described during aging in humans and other species. Symptoms in the lower urinary tract are more prevalent among the elderly. Clinical urodynamic studies have demonstrated that aging is related to reduced bladder capacity, increased uninhibited contractions, decreased urinary flow rate, diminished urethral pressure profile (particularly in women) and increased postvoid residual urine volume (1). In particular, the aging bladder may be described as manifesting detrusor overactivity, impaired contractility or a combination of both.

Several factors have been thought to play a role in the voiding dysfunction in the elderly, including detrusor fibrosis and consequent impairment of contractility (2), deposition and cross-linking of collagen and elastin, and the loss of acetylcholinesterase-positive nerves. The deposition of collagen and fibrosis are recognized phenomena in the aging bladders of both men and women (3). The cause of fibrosis in the aging bladder is unknown. In an animal model, pelvic ischaemia has been shown to induce marked bladder wall fibrosis and loss of compliance (4). Similarly, pelvic ischaemia has been shown to cause detrusor instability in the absence of outflow obstruction (5). Despite these known age-related alterations in detrusor function, the mechanisms of ischaemia and inflammation have not been extensively studied in the human bladder, although 
an overproduction of prostanoids and leukotrienes has been indicated in ischaemic bladder tissue (6).

The vascular endothelial cell growth inhibitor (VEGI) is a multifunctional gene that belongs to the tumour necrosis factor (TNF) superfamily (TNFSF15) and is predominantly expressed in endothelial cells (7). One of three identified VEGI isoforms, VEGI-251 [also known as TNF-like ligand 1A (TL1A)], is the only known ligand for death-domain receptor (DR)3, which is primarily expressed in activated lymphocytes and, to a minor extent, in natural killer cells, macrophages and endothelial cells (8). The interaction of TL1A and DR3 activates a signaling cascade that results in the activation of nuclear factor $-\kappa \mathrm{B}(\mathrm{NF}-\kappa \mathrm{B})$ and the initiation of transcription (7). It has been documented that TL1A inhibits endothelial cell proliferation, angiogenesis and tumour growth (9). In a previous study, we showed that VEGI regulates the tumourrelated vasculature in urothelial tumours of the bladder (10). Both TL1A and DR3 are upregulated in tubular epithelial cells in renal ischaemic injury (11), and they are also involved in several inflammatory diseases, such as Crohn's disease (12), inflammatory bowel disease (13) and rheumatoid arthritis (14). An involvement of TL1A and DR3 in atherosclerosis has also been mentioned (8). TL1A is thus associated with the ischaemic and inflammatory changes in these diseases.

Collectively, these studies imply that TL1A plays a critical role in several inflammatory diseases. However, the role of TL1A in aged bladder dysfunction remains unknown. In the present study, we investigated the TL1A and DR3 expression levels in the aged bladder and explored the possible role of $\mathrm{NF}-\kappa \mathrm{B}$ in ischaemia and inflammation of the aging bladder.

\section{Patients and methods}

Patients. Sixteen bladder tissue samples were obtained from patients with urothelial tumours of the urinary bladder and divided into two groups according to age. The average age of the aged group was $75 \pm 6$ years, while that of the control was $43 \pm 7$ years. None of the patients were found to have bladder outlet obstruction (BOO), as shown by urodynamic assessment. Urodynamic descriptions conformed to the standards of the International Continence Society (ICS) (15). The bladder tissues were obtained at least $5 \mathrm{~cm}$ away from the margin of the tumour tissue and stored at $-80^{\circ} \mathrm{C}$ immediately. The study was approved by the Ethics Committee of the Capital Medical University, and all patients provided informed consent.

Real-time reverse transcription-PCR. For real-time reverse transcription-PCR (RT-PCR) of TL1A, DR3, von Willebrand factor (vWF), nerve growth factor $(\mathrm{NGF})$ and interleukin (IL)-6, total RNA was extracted from the bladder samples using RNeasy ${ }^{\circledR}$ spin columns (Qiagen, Minneapolis, MN, USA) according to the manufacturer's recommendations. DNase digestion was performed for $60 \mathrm{~min}$ to eliminate the contamination of genomic DNA. Fist-strand cDNA was synthesized using the SuperScript ${ }^{\circledR}$ first-strand synthesis system (Invitrogen, Carlsbad, CA, USA). Real-time RT-PCR was performed with power $\mathrm{SYBR}^{\circledR}$-Green PCR Master Mix (ABI, USA) in a 25- $\mu$ l tube with a total reaction volume of $25 \mu \mathrm{l}$ containing $1 \mu \mathrm{l}$ cDNA, $2 \mu \mathrm{l}$ gene-specific upstream and downstream primers. The amplification and analysis of cDNA
Table I. Primer sets for quantitative RT-PCR.

\begin{tabular}{ll}
\hline Primer & \multicolumn{1}{c}{ Sequence (5'-3') } \\
\hline $\begin{array}{l}\text { TL1A } \\
\text { Forward } \\
\text { Reverse }\end{array}$ & CAAACAAGCCAGACTCCATCACT \\
DR3 & GAGAACATGGCTCCGAGGTAGAT \\
Forward & AAGGCGAAGAAGCACGAACGAATG \\
Reverse & ACTCCGGCCGAGAAGTTGAGAAAT \\
vWF & \\
Forward & ATGAGTATGAGTGTGCCTGC \\
Reverse & GTAGATGGTGCTTCGGTGG \\
NGF & \\
Forward & ACAGACATCAAGGGCAAGG \\
Reverse & TGAGTTCCAGTGCTTTGAGTC \\
IL-6 & \\
Forward & CCACTCACCTCTTCAGAACG \\
Reverse & CATCTTTGGAAGGTTCAGGTTG \\
GAPDH & \\
Forward & TGGGTGTGAACCATGAGAAG \\
Reverse & GAGTCCTTCCACGATACCAAAG \\
\hline
\end{tabular}

TL1A, tumour necrosis factor-like ligand 1A; DR3, death-domain receptor 3; vWF, von Willebrand factor; NGF, nerve growth factor; IL-6, interleukin-6.

fragments were managed with a 7300 real-time PCR system (ABI). The mixture was initially heated to $95^{\circ} \mathrm{C}$ for $10 \mathrm{~min}$ to activate hot-start iTaq DNA polymerase and then exposed to 40 cycles of denaturation at $95^{\circ} \mathrm{C}$ for $20 \mathrm{sec}$ and annealing at $60^{\circ} \mathrm{C}$ for $1 \mathrm{~min}$. The amplification of the housekeeping gene, GAPDH, mRNA was used as to normalize the results. The mRNA levels were measured as cycle threshold levels and normalized to the individual GAPDH control cycle threshold values. Sense and antisense primers are shown in Table I.

Western blot analysis. Bladder tissues were grinded and lysed in RIPA buffer containing a cocktail of protease inhibitors (9). The lysate was cleared of cellular debris and the protein concentration was determined with the Bio-Rad DC protein assay (Bio-Rad, Hercules, CA, USA). Samples $(40 \mu \mathrm{g})$ were then loaded onto a $10 \%$ SDS-PAGE gels. Gel-fractionated proteins were transferred to a nitrocellulose membrane. After blocking with $8 \%$ skimmed milk powder in TBST solution, the membranes were blotted with primary antibodies, including TL1A (1:100, LS-B2406; LifeSpan), DR3 (1:500, ab8412; Abcam), and then incubated overnight at $4^{\circ} \mathrm{C}$. The transfers were then rinsed with TBST and incubated with horseradish peroxidase-conjugated anti-rabbit or anti-mouse secondary antibody for $1 \mathrm{~h}$ at room temperature. Immunoblots were visualized on X-ray film (Kodak) with ECL Western Blotting Substrate (Pierce). Developed films were scanned and analyzed with Quantity One 4.62 software. The relative expression levels of TL1A and DR3 proteins were normalized to those of $\beta$-actin (1:2,000; Sigma).

We determined the activation of $\mathrm{NF}-\kappa \mathrm{B}$ by examining the p65 protein expression in the nucleus, and the nucleoproteins were extracted with the Nucleoprotein Protein Extraction kit (BestBio, China). The concentration of primary antibodies 
Table II. Urodynamic parameters of the two groups $(n=16)$.

\begin{tabular}{lccc}
\hline & $\begin{array}{c}\text { Aged } \\
\text { group } \\
(\mathrm{n}=8)\end{array}$ & $\begin{array}{c}\text { Controls } \\
(\mathrm{n}=8)\end{array}$ & P-value \\
Urodynamic parameters & & & \\
\hline Free-flow uroflowmetry & & & \\
Qmax (ml/sec) & $15.7 \pm 2.4^{\mathrm{a}}$ & $20.5 \pm 5.1$ & 0.031 \\
Flow time (sec) & $19.6 \pm 6.4^{\mathrm{a}}$ & $13.8 \pm 3.4$ & 0.044 \\
Voided volume (ml) & $326.0 \pm 73.5$ & $392.0 \pm 61.1$ & 0.068 \\
Residual urine (ml) & $29.2 \pm 11^{\mathrm{a}}$ & $5.7 \pm 6.7$ & 0.000 \\
Pressure-flow study & & & \\
Qmax (ml/sec) & $9.8 \pm 2.5$ & $11.8 \pm 2.6$ & 0.146 \\
Pdet Qmax (cmH & & \\
& $26.5 \pm 5.2^{\mathrm{a}}$ & $33.0 \pm 4.1$ & 0.016 \\
\hline
\end{tabular}

All data are expressed as the means \pm SD. The mean difference was

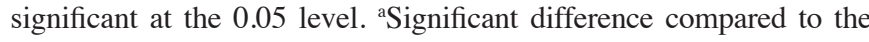
control group.

was 1:1,000 (sc-109; Santa Cruz), and the p65 protein level was normalized to that of histone $\mathrm{H} 3$ (1:1,000, \#9715; Cell Signaling). The remain procedures were identical to those mentioned above.

Statistical analysis. Data are expressed as the means \pm SD and analyzed with the SPSS11.5 software package for Microsoft Windows. Differences between the groups were evaluated by an independent-samples t-test. $\mathrm{P}<0.05$ denoted a statistically significant difference.

\section{Results}

Urodynamic studies. Among the pre-operative urodynamic parameters, the maximum flow rate was decreased, flow time was prolonged, detrusor pressure at maximum flow rate was diminished and residual urine was increased in the aged group compared to the controls $(\mathrm{P}<0.05)$ (Table II).

mRNA expression of TL1A and DR3 in bladder tissues. The mRNA expression levels of TL1A and DR3 were examined in 16 human bladder tissues using real-time RT-PCR. As shown in Fig. 1A, the aged bladder tissues displayed increased expression levels of TL1A and DR3, compared to the control group $(\mathrm{P}<0.05$ and $\mathrm{P}<0.01$, respectively).

mRNA expression of $v W F, I L-6$ and NGF in bladder tissues. As shown in Fig. 1B, there was a significant downregulation of $\mathrm{vWF}$ in the aged bladders compared to the control group $(\mathrm{P}<0.01)$. The IL-6 mRNA expression levels of the aged group were significantly higher than those of the control group $(\mathrm{P}<0.01)$. Although the expression levels of NGF mRNA in the aged group were lower than those of the controls, no significance was detected between the two groups.

Protein expression of TL1A and DR3 is upregulated in the aged bladder tissues. The relative expression levels of TL1A and DR3 proteins were normalized to those of $\beta$-actin. As shown in Fig. 2A, the aged group exhibited increased expression
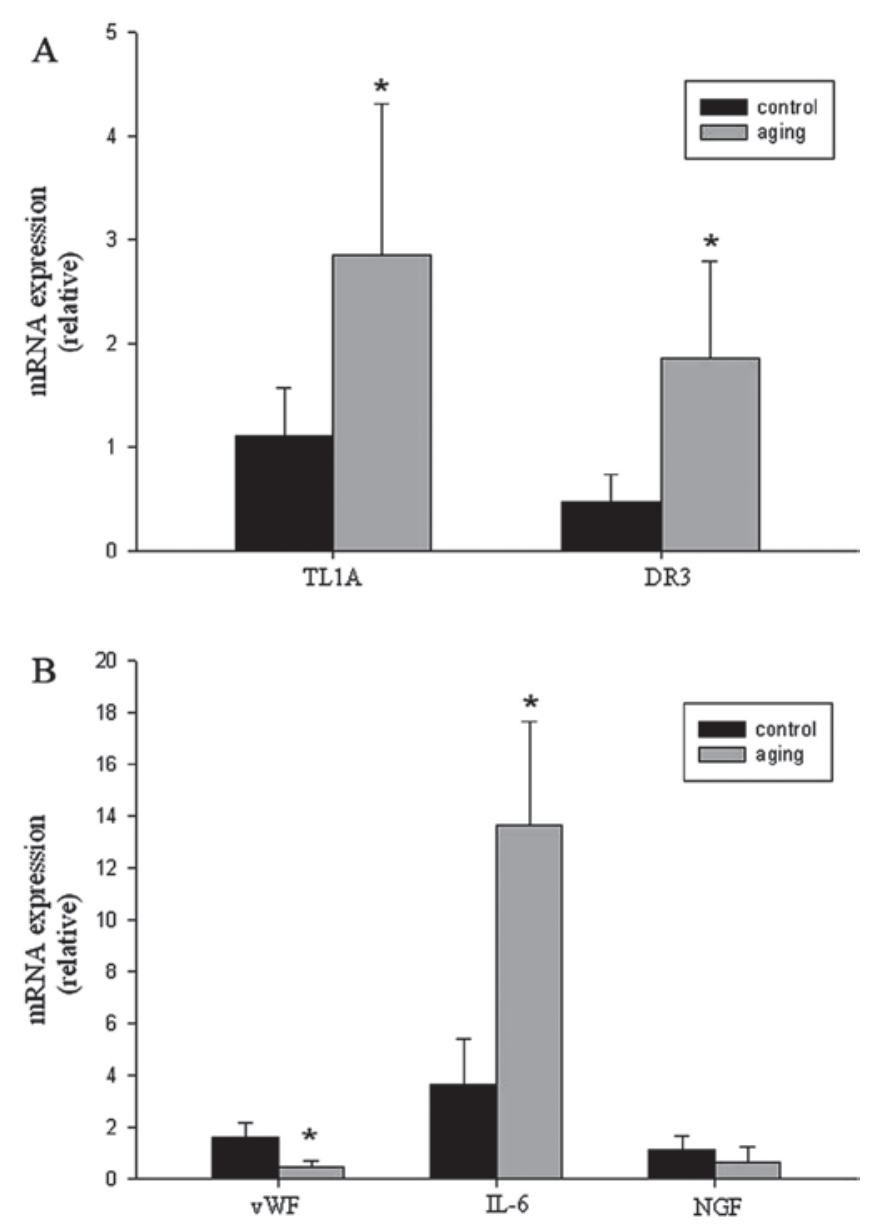

Figure 1. mRNA expression of TL1A, DR3, vWF, IL-6 and NGF. The mRNA expression was examined using real-time RT-PCR. (A) mRNA expression of TL1A and DR3 was increased in aged bladder tissue. (B) There was a decrease in the expression of $\mathrm{vWF}$ in aged bladders as compared to the controls. However, the mRNA expression of IL-6 was increased in aged bladder tissue compared to control. There was no difference in the mRNA expression of NGF between the aged group and the control. ${ }^{*} \mathrm{P}<0.05$. TL1A, tumour necrosis factor-like ligand 1A; DR3, death-domain receptor 3; vWF, von Willebrand factor; NGF, nerve growth factor; IL-6, interleukin-6.

levels of both TL1A and DR3 compared to the control group $(\mathrm{P}<0.05)$ (Fig. 2B).

$N F-\kappa B$ is activated in aged bladder tissues. The activation of $\mathrm{NF}-\kappa \mathrm{B}$ requires the dissociation of its p65 and p50 subunits, from an inhibitory subunit known as the inhibitor of $\mathrm{\kappa B}$ ( $\mathrm{I} \kappa \mathrm{B} \alpha$ ), which is accomplished by the phosphorylation, ubiquitination and subsequent degradation of I $\mathrm{B} \alpha$. The $\mathrm{p} 65$ subunit of NF- $\mathrm{KB}$ is then translocated into the nucleus where it binds to specific DNA target sites. It was found that the p65 protein expression levels in the nucleoprotein of the aged bladder tissues were significantly upregulated compared those of the control group $(\mathrm{P}<0.01)$ (Fig. 2C).

\section{Discussion}

Although lower urinary tract symptoms (LUTS) are common in the elderly, few studies have focused on elucidating the pathophysiological mechanisms underlying symptoms in aged bladder. Even when studies are conducted, it is difficult to differentiate between purely age-related changes and 
A

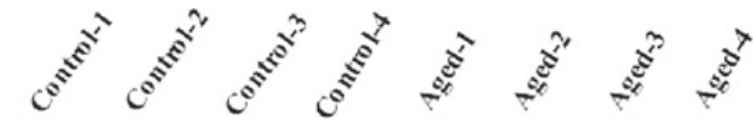

TL1A

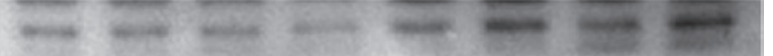

$\beta$-actin

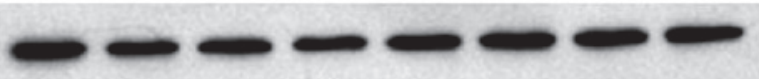

B

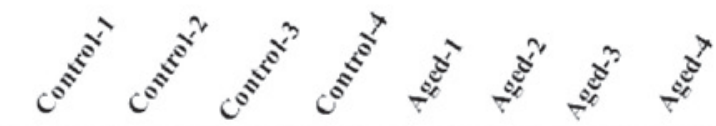

DR3

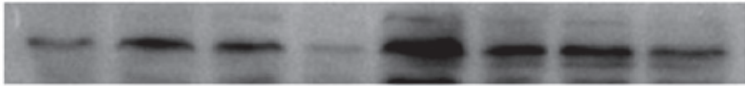

$\beta$-actin

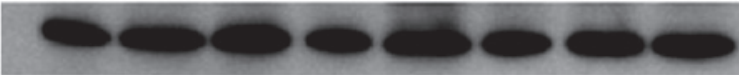

C

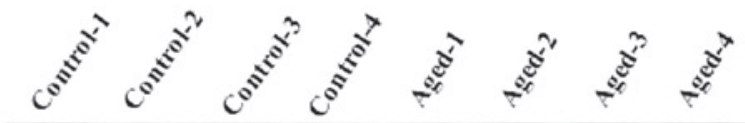

P-65

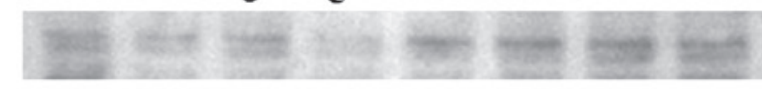

Histone
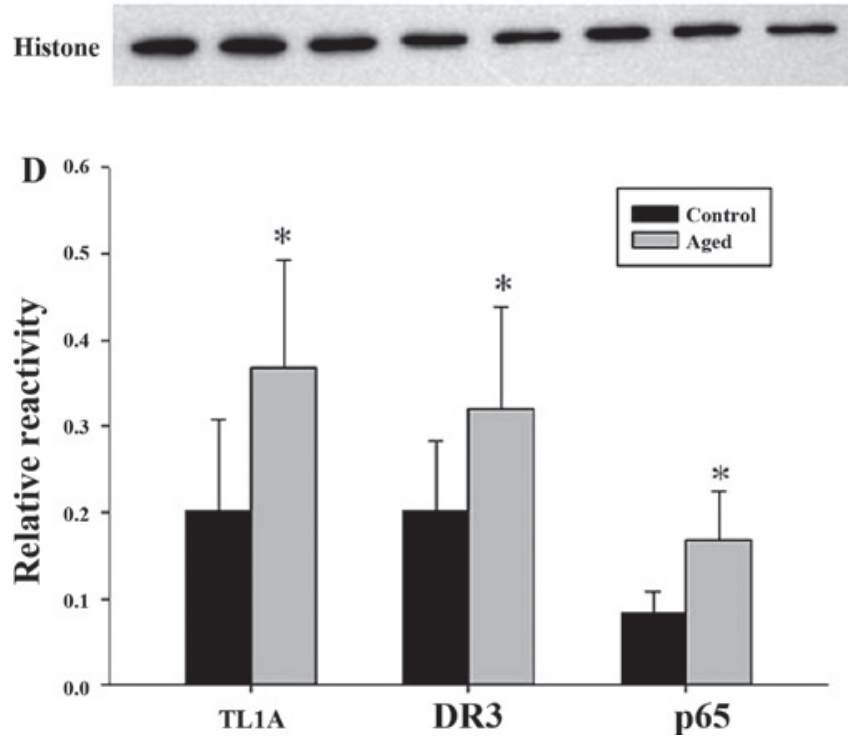

Figure 2. (A) The protein expression of TL1A was increased in the aged group compared to the control. (B) There was a significantly increased DR3 protein expression in the aged group compared to the control. (C) We determined the activation of nuclear factor $-\kappa \mathrm{B}(\mathrm{NF}-\kappa \mathrm{B})$ by examining the $\mathrm{p} 65$ protein expression in the nucleus. The p65 protein expression levels in the nucleoprotein of aged bladder tissue were significantly upregulated compared to the control. (D) Relative protein expression levels normalized to those of $\beta$-actin and histone $\mathrm{H} 3$. $^{*} \mathrm{P}<0.05$. TL1A, tumour necrosis factor-like ligand 1A; DR3, death-domain receptor 3

dysfunction due to disease, such as BOO caused by benign prostatic hyperplasia. Clinically, it is difficult to obtain aging bladder tissue. In the present study, we selected the aged and non-aged patients suffering from urothelial tumours of the bladder. None of the patients were found to have BOO, as shown by urodynamic assessment. In order to avoid the impact of the tumour, the full-thickness bladder tissues were obtianed at least $5 \mathrm{~cm}$ away from the margin of the tumour tissues. In the present study, we analyzed the possible role of TL1A and DR3 and other factors in the ischaemic and inflammatory process of aged bladder dysfunction.
It has previously been reported that chronic bladder ischaemia may play an important role in voiding dysfunction of the aging bladder (16). vWF is a vascular endothelial cell marker, frequently used in the evaluation of microvessel density (MVD) (17). Jahroudi and Lynch revealed an endothelial cellspecific regulation of vWF mRNA expression in vitro (18). Our study showed a decrease of vascular endothelial cell-related gene (vWF) in the aged bladder. This indicates that the aged bladder is likely associated with the reduction in MVD and hence ischaemia, compared to the controls. This is in agreement with previously published data (3). However, the reasons leading to ischaemia are multifold, including arterial disease and hypercholesterolemia. Our study displayed an upregulation of both TL1A and DR3 in aged bladder tissues at the mRNA and protein levels. TL1A has been shown to be an endogenous inhibitor of angiogenesis produced mainly by vascular endothelial cells, and exerts a specific inhibitory activity on the proliferation of endothelial cells (19). TL1A enforces the growth arrest of endothelial cells in the G0 and early G1 phases of the cell cycle, but induces apoptosis in proliferating endothelial cells. Chew et al reported that the overexpression of TL1A induces apoptosis in endothelial cells, resulting in a reduction in MVD (20). TL1A has been found to be significantly upregulated in chronically ischaemic myocardial tissue (21). TL1A mRNA has otherwise been shown to be upregulated in human circulating endothelial progenitor cells from old vs. young human volunteers (22).

In the present study, we show that IL- 6 mRNA expression is upregulated in the aged bladder. IL- 6 is one of the central players in the regulation of inflammation and immune reactions. The interaction between the members of the TNF superfamily and their receptors (e.g., TNF- $\alpha$ ) elicit diverse biological responses, which are known to be involved in post-ischaemic inflammation (23). Previous studies have shown that inflammatory cell infiltration is possibly involved in the aged bladder (24). The cause of the inflammatory reaction is unknown; however, the failure to detect a causative microorganism suggests that it may be an autoimmune response. In addition, a number of studies have suggested that ligand-receptor interactions of TL1A and DR3 are involved in the development of diverse T cell-mediated autoimmune diseases (7). Early reports from in vitro functional studies have indicated that TL1A enhances T cell proliferative responses and augments Th1 cytokine production, such as interferon- $\gamma$ and IL-6. Upregulated TL1A results in intestinal fibrosis in the mouse inflammatory bowel disease model. It is known that the binding of TL1A and DR3 activates the NF- $\kappa$ B pathway (25). Therefore, we hypothesized that the interaction between TL1A and DR3 is involved in the process of bladder inflammation.

The activation of $\mathrm{NF}-\kappa \mathrm{B}$ is one of the events triggered by various members of the TNF family. This activation requires the dissociation of its cytoplasmic heterodimer, composed of the p50 and p65 subunits, and the p65 subunit translocates into the nucleus to bind to specific sites on DNA. In our study, we demonstrate an overexpression of the p65 protein in the aged bladder tissue. As the increased activation of NF- $\kappa \mathrm{B}$ leads to the enhanced expression of these pro-inflammatory mediators, the NF- $\mathrm{NB}$ activation may be a central event in the development of multiple organ dysfunction associated with infection, blood loss and ischaemia-reperfusion injury. 
The ischaemia-induced detrusor overactivity involves bladder innervation, muscarinic receptors and the epithelium, and increases prostaglandin and leukotriene production. Human detrusor biopsies reveal axonal degeneration, muscle loss and fibrosis in detrusor underactivity. Bladder neural reactions to ischaemia may involve multiple mechanisms. Neurodegeneration has been documented in human neurogenic bladder dysfunction (26). NGF is a secretory protein which plays a critical role in the development of the peripheral nervous system. NGF has been shown to act positively on axonal regeneration and outgrowth, and partially reverse neuropathy (27). The function of NGF to protect nerve fibers against ischaemia/reperfusion brain injury has been documented (28). Our RT-PCR data showed that the NGF mRNA expression in the aged bladder tissues was lower than that in the control, although this difference did not reach statistical significance. It has been reported that the urinary levels of NGF are significantly increased in patients with an overactive bladder (29). However, there has also been a report showing that NGF is not significantly associated with detrusor overactivity (30). We argue that the difference of NGF expression levels in aged patients may be related to detrustor overactivity or underactivity.

Finally, although the present study shows that TL1A expression is upregulated in the aged bladder and involved in the process of aged bladder dysfunction, we were unable to determine the cell and tissue location of TL1A expression (mucosal or detrusor cells). In future studies, we will explore the spatial distribution of both TL1A and DR3 in aging bladder tissues and further explore, at cellular and organ levels, the mode of action of TL1A in this condition.

In conclusion, the expression levels of TL1A and DR3 are upregulated in aged bladders, together with an increased expression of IL- 6 and a decreased expression of vWF mRNA. This suggests that the ischaemia and inflammation of aged bladder tissues result in bladder dysfunction, in which TL1A and DR3 play an important role.

\section{Acknowledgements}

This study was supported by the Beijing Natural Science Foundation, grant no. 7102061 .

\section{References}

1. Madersbacher S, Pycha A, Schatzl G, Mian C, Klingler CH and Marberger M: The aging lower urinary tract: a comparative urodynamic study of men and women. Urology 51: 206-212, 1998

2. Taylor JA III and Kuchel GA: Detrusor underactivity: clinical features and pathogenesis of an underdiagnosed geriatric condition. J Am Geriatr Soc 54: 1920-1932, 2006.

3. Azadzoi KM, Tarcan T, Siroky MB and Krane RJ: Atherosclerosisinduced chronic ischaemia causes bladder fibrosis and non-compliance in the rabbit. J Urol 161: 1626-1635, 1999.

4. Azadzoi KM, Radisavljevic ZM, Golabek T, Yalla SV and Siroky MB: Oxidative modification of mitochondrial integrity and nerve fiber density in the ischaemic overactive bladder. J Urol 83: 362-369, 2010

5. Azadzoi KM, Tarcan T, Kozlowski R, Krane RJ and Siroky MB Overactivity and structural changes in the chronically ischaemic bladder. J Urol 162: 1768-1778, 1999.

6. Azadzoi KM, Shinde VM, Tarcan T, Kozlowski R and Siroky MB: Increased leukotriene and prostaglandin release, and overactivity in the chronically ischaemic bladder. J Urol 169: $1885-1891,2003$
7. Migone TS, Zhang J, Luo X, et al: TL1A is a TNF-like ligand for DR3 and TR6/DcR3 and functions as a T cell costimulator. Immunity 16: 479-492, 2002.

8. Kang YJ, Kim WJ, Bae HU, et al: Involvement of TL1A and DR3 in induction of pro-inflammatory cytokines and matrix metalloproteinase-9 in atherogenesis. Cytokine 29: 229-235, 2005.

9. Zhai Y, Yu J, Iruela-Arispe L, et al: Inhibition of angiogenesis and breast cancer xenograft tumour growth by VEGI, a novel cytokine of the TNF superfamily. Int J Cancer 82: 131-136, 1999.

10. Zhang N, Sanders AJ, Ye L, Kynaston HG and Jiang WG: Expression of vascular endothelial growth inhibitor (VEGI) in human urothelial cancer of the bladder and its effects on the adhesion and migration of bladder cancer cells in vitro. Anticancer Res 30: 87-95, 2010.

11. Al-Lamki RS, Wang J, Tolkovsky AM, et al: TL1A both promotes and protects from renal inflammation and injury. J Am Soc Nephrol 19: 953-960, 2008.

12. Takedatsu H, Michelsen KS, Wei B, et al: TL1A (TNFSF15) regulates the development of chronic colitis by modulating both T-helper 1 and T-helper 17 activation. Gastroenterology 135: 552-567, 2008.

13. Bamias G, Martin C III, Marini M, et al: Expression, localization, and functional activity of TL1A, a novel Th1-polarizing cytokine in inflammatory bowel disease. J Immunol 171: 4868-4874, 2003.

14. Zhang J, Wang X, Fahmi H, et al: Role of TL1A in the pathogenesis of rheumatoid arthritis. J Immunol 183: 5350-5357, 2009

15. Abrams P, Blaivas JG, Stanton SL and Andersen JT: The standardisation of terminology of lower urinary tract function. The International Continence Society Committee on Standardisation of Terminology. Scand J Urol Nephrol Suppl 114: 5-19, 1988.

16. Azadzoi KM, Yalla SV and Siroky MB: Oxidative stress and neurodegeneration in the ischaemic overactive bladder. J Urol 178: 710-715, 2007.

17. Poncelet C, Madelenat P, Feldmann G, Walker F and Darai E: Expression of von Willebrand's factor, CD34, CD31, and vascular endothelial growth factor in uterine leiomyomas. Fertil Steril 78: 581-586, 2002.

18. Jahroudi N and Lynch DC: Endothelial-cell-specific regulation of von Willebrand factor gene expression. Mol Cell Biol 14: 999-1008, 1994.

19. Tian F, Liang PH and Li LY: Inhibition of endothelial progenitor cell differentiation by VEGI. Blood 113: 5352-5360, 2009.

20. Chew LJ, Pan H, Yu J, et al: A novel secreted splice variant of vascular endothelial cell growth inhibitor. FASEB J 16: 742-744, 2002.

21. Lachtermacher S, Esporcatte BL, Montalvao F, et al: Cardiac gene expression and systemic cytokine profile are complementary in a murine model of post-ischaemic heart failure. Braz $\mathrm{J}$ Med Biol Res 43: 377-389, 2010.

22. Shih DQ, Kwan LY, Chavez V, et al: Microbial induction of inflammatory bowel disease associated gene TL1A (TNFSF15) in antigen presenting cells. Eur J Immunol 39: 3239-3250, 2009.

23. Gu Q, Yang XP, Bonde P, DiPaula A, Fox-Talbot K and Becker LC: Inhibition of TNF-alpha reduces myocardial injury and proinflammatory pathways following ischaemia-reperfusion in the dog. J Cardiovasc Pharmacol 48: 320-328, 2006.

24. Phillips JI: Inflammatory plasma cell infiltration of the urinary bladder in the aging C57BL/Icrfa(t) mouse. Invest Urol 19: 75-78, 1981.

25. Wen L, Zhuang L, Luo X and Wei P: TL1A-induced NF-kappaB activation and c-IAP 2 production prevent DR3-mediated apoptosis in TF-1 cells. J Biol Chem 278: 39251-39258, 2003.

26. Haferkamp A, Dorsam J, Resnick NM, Yalla SV and Elbadawi A Structural basis of neurogenic bladder dysfunction. III. Intrinsic detrusor innervation. J Urol 169: 555-562, 2003.

27. Karamoysoyli E, Burnand RC, Tomlinson DR and Gardiner NJ: Neuritin mediates nerve growth factor-induced axonal regeneration and is deficient in experimental diabetic neuropathy. Diabetes 57: 181-189, 2008

28. Yang JP, Liu HJ, Yang H and Feng PY: Therapeutic time window for the neuroprotective effects of NGF when administered after focal cerebral ischaemia. Neurol Sci 32: 433-441, 2011.

29. Lowe EM, Anand P, Terenghi G, Williams-Chestnut RE, Sinicropi DV and Osborne JL: Increased nerve growth factor levels in the urinary bladder of women with idiopathic sensory urgency and interstitial cystitis. Br J Urol 79: 572-577, 1997.

30. Birder LA, Wolf-Johnston A, Griffiths D and Resnick NM: Role of urothelial nerve growth factor in human bladder function. Neurourol Urodyn 26: 405-409, 2007. 